\title{
How's going on the T-Cell Brazil project
}

\begin{abstract}
Lymphomas are a large, heterogeneous and infrequent group of neoplasms. Peripheral t-cell lymphomas (PTCL) is still rarer, and it makes up about $10-15 \%$ of lymphoid malignancies. Its prognosis is poor and remains a challenge. In April 2017, the first Brazilian collaborative effort was created to collect data from T-cell Lymphoma patients diagnosed in the five distinct macro-regions of Brazil. To better understand PTCL, according to the revised WHO-2017 classification, excluding Mycosis fungoides, Sézary syndrome, and chronic Lymphoproliferative disorders, all histological subtypes diagnosed from January 2015 to December 2022 will be enrolled. Tissue biopsied, immunophenotypic markers from consecutive patients at each site will be reviewed by panels of expert hematopathologists every year. So far, 381 cases were enrolled by 32 Brazilian centers, most of them are from the Southeast and South region. Twenty-two cases were not evaluable due to lack of complete data; hence, 359 were analyzed. The overall survival at 24 -month was $52 \%$ (95\% CI: $46-58 \%$ ) and progression-free survival 37\% (95\% CI: $31-43 \%)$ with a median time of follow-up of 10 months (0.2-64). T-cell Brazil Project has many challenges to overcome, mainly due to its vast territory and its disparity. However, we already have another sixteen centers waiting for approval in their Ethics Committees. It was created a network around the country, mainly among the pathologists, with educational goals and exchange of experiences. It is crucial to obtain knowledge of local epidemiology to optimize resources, design clinical trials, and identify minority entities, contributing to increasing the quality of the diagnosis in T-cell Lymphomas, consequently improving their treatments and quality of life.
\end{abstract}

Keywords: Peripheral t-cell lymphomas (PTCL), overall survival, T-cell Brazil Project
Volume 9 Issue 3 - 202I

\author{
Carmino De Souza,' Marcia T Delamain, ${ }^{2}$ \\ Eliana C M Miranda, ${ }^{2}$ Juliana Pereira, ${ }^{3}$ \\ Danielle Leão, ${ }^{4}$ Sergio A B Brasil, ${ }^{5}$ Nelson $S$ \\ Castro, ${ }^{6}$ Flavia F S Zacchi, ${ }^{7}$ Tomas Z Barrese, ${ }^{8}$ \\ José Vassalo, ${ }^{9}$ Carlos Chiattone ${ }^{5,10}$ \\ 'Medical Sciences Faculty of University of Campinas, Brazil \\ ${ }^{2}$ Hematology and Hemotherapy Center of University of \\ Campinas, Brazil \\ ${ }^{3}$ Laboratory of Medical Investigation in Pathogenesis and \\ Directed Therapy in Onco-Immuno-Hematology, Medicine \\ School of University of São Paulo, Brazil \\ ${ }^{4}$ Beneficiencia Portuguesa Hospital of Sao Paulo, Brazil \\ ${ }^{5}$ Santa Casa Medical School, Brazil \\ ${ }^{6}$ Fundação Pio XII, Cancer Hospital of Barretos, Brazil \\ ${ }^{7}$ Grupo Fleury Sao Paulo, Brazil \\ ${ }^{8}$ Grupo Fleury Sao Paulo and Adolfo Lutz Institute, Brazil \\ 'Laboratory of Investigative and Molecular Pathology, CIPED, \\ Medical Sciences Faculty of University of Campinas, Brazil \\ ${ }^{10}$ Samaritano Hospital, SP, Brazil
}

Correspondence: Carlos Chiattone, Santa Casa Medical School, Samaritano Hospital, Sao Paulo, Brazil, Email carlos.chiattone@terra.com.br

Received: May 06, 202I | Published: May 2I, 202 I

\section{Introduction}

Lymphomas are a large, heterogeneous, rare group of neoplasms, and PTCL is still more infrequent; it makes up about $10-15 \%$ of lymphoid malignancies. Currently, the revised WHO 2017 criteria sub-classified PTCL with more than 20 entities. The most frequent subtype is PTCL-NOS (not otherwise specified); it is a term when features do not fit known entities established by the revised 2017 WHO classification. Its prognosis is poor and remains a challenge.

The Brazilian National Cancer Institute (INCA) ${ }^{2}$ estimated 12,030 new cases of all NHL, being the estimated risk of new cases by year of 5.70/100,000 habitants in 2020. In Brazil, the literature is scarce regarding the clinical and epidemiological data of PTCL, and its heterogeneity already justifies the importance of obtaining data representing the real-world situation. Besides that, Brazil has a highly heterogeneous climate, geography, and environment, having a great genomic diversity. The Brazilian population has over 212 million people distributed unequally by different regions that have unique particularities in many aspects. ${ }^{3}$

Recognizing this immense disparity and knowing that these data may affect the epidemiology of the disease, reflecting even in the diagnosis, treatment, and results, it was decided to face the challenge of developing a Brazilian registry of PTCL, mainly due to the lack of information on the profile of this disease in the country. So, on April 1, 2017 to collect a cohort of at least 500 patients diagnosed with PTCL was opened the T-Cell Brazil Project. ${ }^{4}$

\section{Methods}

This study was designed as an ambispective data collection with the diagnosis made from January 2015 to December 2022.
Participating Institutions are also asked to provide a consecutive series of cases, without any selection, once permission is obtained from the Institutional Review Board (IRB) as required by individual, institutional policies.

Inclusion criteria were previously untreated patients diagnosed with PTCT or NK/T-cell lymphoma according to the revised WHO 2017 classification, excluding mycosis fungoides, Sézary syndrome and chronic lymphoproliferative disorders; age over 18; tissue biopsies adequate for diagnosis, classification and available for centralized review; clinical and laboratory data, features of treatment chosen and assurance of follow-up for at least five years for living patients.

Patient registrations are performed using the REDcap ${ }^{5}$ (Research Electronic Data Capture tools) hosted at the Hematology and Hemotherapy Center at the University of Campinas, accessible to each investigator by requesting a personal login. REDcap Platform adopts SSL03 technology assuring protection in web communications of the subject's clinical data obeying the Brazilian data protection laws.

Overall survival (OS) was defined as the time from diagnosis to death or last seen, and the event was death. Progression-free survival (PFS) was defined as the time from diagnosis to relapse/progression or death from any cause or latest follow-up, and its event was relapse/ progression or death. The data were analyzed using descriptive tools, Kaplan-Meier method, and log-rank test, considering significant P-value $<5 \%$, using SPSS (Statistic Package Social Sciences) v.24.

\section{Preliminary results}

T-cell Brazil Project has registered 381 patients until March 31, 2021. However, 359 (94\%) were analyzed, and 22 (6\%) were excluded from this analysis due to lack of complete data. The median 
age was 54 years (18-92), 58\% of patients were male, and advancedstage disease (Ann Arbor stage III-IV) was found in $72 \%$ and ECOG (Eastern Cooperative Oncology Group) $\geq 1$ around 64\%.

The distribution of subtypes was PTCL-NOS, 31\%, anaplastic large-cell lymphoma ALK- (ALCL ALK-) 17.6\%, Adult T-cell Leukemia/Lymphoma (ATLL) 14\%, Extranodal NK/T cell, nasal type (ENKTL) 13\%, Angioimmunoblastic T-cell lymphoma (AITL) $12.3 \%$, ALCL ALK+ 7\%, large granular lymphocyte (LGL) 1.8\%, hepatosplenic t-cell lymphoma, (HSTCL) 1.2\%, subcutaneous panniculitis-like t-cell lymphoma (SPTCL) $0.6 \%$, enteropathy type t-cell lymphoma (EATL) $0.6 \%$, breast implant-associated ALCL (BIA-ALCL) $0.6 \%$ and Aggressive NK-cell leukemia $0.3 \%$.

Chemotherapy was used in 319 (89\%) patients being the most frequent CHOEP (47\%) (cyclophosphamide, doxorubicin, vincristine, prednisone plus etoposide) and CHOP (20\%) (cyclophosphamide, doxorubicin, vincristine and prednisone). It was used 4\% AZT plus interferon, $3.5 \%$ corticoids, $2 \%$ interferon alone and $1.5 \%$ Brentuximab. Radiotherapy was applied in 47 (13\%) patients, and the transplant as consolidation occurred in 50 cases (14\%) cases.

Progression diseases occurred 115 cases (32\%) and $142(39.5 \%)$ deaths, being 43 early deaths before completing the first treatment. The causes of death were 95 (67\%) lymphoma, 33 (23.2\%) infections; $9(6.3 \%)$ toxicities; one $(0.7 \%)$ new neoplasia and $4(2.8 \%)$ undetermined ( 3 cases in complete remission and one stable disease). At a median follow-up of 10 months $(0.2-64)$, overall survival at 24 -month was $52 \%(95 \% \mathrm{CI}: 46-58)$, whereas progression-free survival was $37 \%$ (95\% CI: 31-43) (Table 1).

Table I Baseline Demographic and Clinical Features

\begin{tabular}{|c|c|}
\hline Variable & $n=359$ \\
\hline Gender= male, $\mathrm{n}(\%)$ & $220(58.5 \%)$ \\
\hline Median age (range) & $54(18-92)$ \\
\hline Ann Arbor Stage & $345 / 359(96 \%)$ \\
\hline$|A /| B$ & $31 / 17$ (14\%) \\
\hline$\|A /\| B$ & $29 / 20$ (14.5\%) \\
\hline III A / III B & $23 / 44$ (19.5\%) \\
\hline IV A / IV B & 49 / I 32 (52\%) \\
\hline ECOG / Performance Status $\geq 1$ & 227 / 353 (64\%) \\
\hline Lymph nodes involvement & $247 / 353(70 \%)$ \\
\hline Extra nodal involvement & $195 / 351(55.5 \%)$ \\
\hline Bone Marrow involvement & $100 / 354(28 \%)$ \\
\hline CNS involvement & $13 / 35 \mid(3.5 \%)$ \\
\hline HIV+ serology & II /347 (3\%) \\
\hline HTLV+ serology & $53 / 347$ (15\%) \\
\hline Final status: death & I42/359 (39.5\%) \\
\hline Median of follow-up, months (range) & $10(10-64)$ \\
\hline
\end{tabular}

ECOG, eastern cooperative oncology group; CNS, central nervous system

\section{Discussion}

Although these are very preliminary data, T-cell Brazil Project has shown that frequencies of the main PTCL subtypes have some differences as expected by the literature. ${ }^{6}$ The PTCL-NOS was the most frequent, followed by ALCL-ALK negative. Remarkable, at this time, we see a higher frequency of ATLL and ENKTL, probably due to the inclusion of all different Brazilian regions.

Related to treatment, T-cell Brazil project ${ }^{4}$ reflects the patients' outcomes treated according to standards of care in Brazil. Despite their heterogeneity, the vast majority of PTCLs are treated similarly, using upfront anthracycline-based therapy, likewise as mentioned by international centers. ${ }^{7}$

Only $14 \%$ of our patients underwent to autologous transplant as a consolidation after CR or PR. We are looking for a data which can explain the reasons for that.

Among challenges present in this project, one is to pick up more cases from North, Northeast, Central-West, and South Centers. Then, we will confirm the epidemiological differences found in several countries from Latin America compared to North America, Europe, and Asia countries. ${ }^{6}$

In conclusion, our data are preliminaries, but up to now it was possible to construct a network around the country, mainly among the pathologists, with educational goals and exchange of experiences, contributing for increasing the quality of the diagnosis in T-cell Lymphomas. We are reaching the objective of building a registry representative of the Brazilian population, including patients of all the five regions of the country. This project highlights the importance of multicenter cooperation, which should be mandatory when studies are focused on rare diseases.

\section{Contributors}

All authors contributed to drafting this manuscript, with CSC taking a lead role. All authors provided intellectual input to improve the manuscript and have read and approved the final version. CSC is the guarantor of the manuscript.

\section{Funding}

The authors have not declared a specific grant for this research from any funding agency in public, commercial or not-for-profit sectors.

\section{Ethics approval and consent to participate}

All procedures explained in the paper were in accordance with the ethical standards of the University of the Social Welfare and rehabilitation research committee and with the 1964 Helsinki Declaration. All information anonymously collected and received participants contest.

\section{Acknowledgments}

Educational Support by Takeda.

\section{Conflicts of interest}

The authors declare no conflicts of interest.

\section{Trial investigators}

Cecyn, KZ (Federal University of Sao Paulo, SP, Brazil); Bellesso, M (HemoMed Institute, SP); Lyrio, R (State University of Rio de Janeiro, UERJ, Brazil); Cunha-Junior, AD (Hematology and Oncology Clinics, Cancer Hospital of Cascavel, União Oeste de Estudos e Combate ao Câncer (UOPECCAN), Cascavel, PR, Brazil); 
Gonzaga, Y (National Institute of Cancer - INCA, RJ, Brazil); Nabhan, S (Federal University of Paraná, PR, Brazil); Zing, N (Prevent Senior Hospital, SP, Brazil); Ribeiro, G (Federal University of Minas Gerais, MG, Brazil); Carneiro, TX (Ophir Loyola Hospital, PA, Brazil); Nogueira, DS (Hospital do Servidor Publico IAMSPE, SP, Brazil); Salvino, MA e Dias, M (Federal University of Bahia, Brazil); SoutoFilho, JTD (Medical Faculty of Campos dos Goytacases, RJ, Brazil); Schaffell, R (Federal University of Rio de Janeiro, Brazil); Dal Pont, M (Centro de Pesquisas Oncológicas (CEPON), Florianópolis, Brazil); Hamerschlak, N (Albert Einstein Hospital, SP, Brazil); Gaiolla, R (Hospital das Clínicas da Faculdade de Medicina de Botucatu (HC-FMB), São Paulo, SP, Brazil); Duarte, FB and Rolim, RR (Federal University of Ceara, School Hospital Walter Cantidio, CE, Brazil); Hallack-Neto, A (Federal University of Juiz de Fora, MG, Brazil); Rabelo, YS (Federal University of Goiás, Goiania, Brazil); Mo, SKG and Pinotti, C (Samaritano Hospital - Higienopolis, SP, Brazil); Negreiros, E (Hospital of Love, Fundação Pio XII, RO, Brazil); Macedo, CCG and Vilarim, CC (Liga Norte Rio Grandense contra Cancer, Natal, RN); Cordeiro, A (AC Camargo Cancer Center, Sao Paulo, Brazil); Radtke-Giacon, PP (Santa Marcelina Hospital, SP, Brazil); Ribeiro, EFO (Santa Lucia Hospital, Brasilia, Brazil); Bueno, ND (Clinica Medica Frad, SP, Brazil).

\section{References}

1. Matutes E. The 2017 WHO update on mature T- and natural killer (NK) cell neoplasms. Int J Lab Hem. 2018;40(Suppl. 1):97-103.

2. Estimativa 2020: incidência de câncer no Brasil / National Cancer Institute of José Alencar Gomes da Silva. Rio de Janeiro: INCA, 2019.

3. Brazil. Ministry of Planning, Budget and Management. Brazilian Institute of Geography and Statistics. Populational Count.

4. Delamain MT, Miranda ECM, Vassallo J, et al. T cell Brazil Project: a Pioneer Project to collect data of T-cell NHL patients among five regions of Brazil. Int Clin Med. 2018:2(2):1-2.

5. Harris PA, Taylor R, Minor BL, et al. The REDCap consortium: Building an international community of software platform partners. $J$ Biomed Inform. 2019;95:103208.

6. Bellei M, Chiattone CS, Luminari S, et al. T-Cell Lymphomas in South America and Europe. Rev Bras Hematol Hemoter. 2012;34(1):42-47.

7. Moskowitz AJ, Lunning MA, Horwitz SM. How I treat the peripheral T-cell lymphomas. Blood. 2014;123(17):2636-2644.

8. Reimer P, Rudiger T, Geissinger E, et al. Autologous stem-cell transplantation as first-line therapy in peripheral T-cell Lymphomas: results of a prospective multicenter study. J Clin Oncol. 2009;27(1):106113. 